\title{
Optic disc drusens mimicking papilloedema
}

\author{
Nishtha Yadavin, Neeti Gupta ${ }^{2, *}$, Amit Maitreya ${ }^{3}$, Renu Dhasmana ${ }^{4}$, Harsh Bahadur ${ }^{5}$ \\ ${ }^{1}$ Resident, ${ }^{2,3}$ Associate Professor, ${ }^{4,5}$ Professor, Dept. of Ophthalmology, Himalayan Institute of Medical Sciences (SRH \\ University), Dehradun, Uttarakhand, India
}

*Corresponding Author:

Email: drneetigupta@rediffmail.com

\begin{abstract}
Optic disc drusens and papilledema have similar fundus presentation, leading to confusion between the two.

Case Report: Here we present a case of 12 years old male who presented with complaints of headache since 1 year associated with nausea and vomiting and was misdiagnosed as papilledema.

Result: Differentiating optic disc drusens from true optic disc edema is critically important, because optic disc edema mostly present as a life-threatening condition requiring urgent and costly ancillary testing, whereas optic disc drusens is often a benign process requiring only observation.

Conclusion: Swelling of the optic discs does not always mean raised intracranial pressure, hence other causes must be considered before concluding.
\end{abstract}

Keywords: Optic disc drusens, Papilloedema.

\section{Introduction}

Papilledema portrays an acute, severe, and lifethreatening condition. When presented with the physical examination finding, it is not to be taken lightly. The differential diagnosis of a papilledema patient includes etiologies from either increased intracranial pressure or from optic disc swelling. ${ }^{1}$ So it has become important to confirm the diagnosis of papilledema, to avoid unnecessary interventations like lumbar puncture, CT or MRI. Optic nerve head drusen presents clinically as pseudo-papilledema. They are calcified lesions elevating a portion of the optic nerve head and thus mimicking papilledema on funduscopic examination.

The prevalence of drusen is around $0.34 \%$, and is estimated to be $3.4 \%$ in individuals with positive family history. It is bilateral in $85 \%$ of population. The most common associated complication is peripheral visual field defects which occurs in approximately $75 \%$ of patients. $^{2}$

\section{Case Report}

A 12 year old male presented to our eye OPD with chief complaint of headache associated with nausea and vomiting since last 1 year, although denied visual changes. Diagnosis of benign intracranial hypertension was made elsewhere and was prescribed oral acetazolamide for 1 year. The cerebrospinal fluid pressure was normal and so he was referred from neurology OPD to rule out pseudo-papilledema. He had no known allergies, and denied illicit drug use. Physical examination revealed a well-developed teenage male in no acute distress. The patient was afebrile, and normotensive.

Ocular examination was normal for both eyes. His ophthalmologic examination revealed bilateral full cup, blurred disc margins consistent with papilledema and spontaneous venous pulsations were present. Rest of the ocular examination was unremarkable.

MRI head of the patient was already done and was suggestive of normal study. $6,7)$

120-2 visual fields were normal for both eye. (Fig.

B SCAN showed hyper-echoic mass at the optic nerve head in both eyes even at low gain, suggestive of optic nerve head drusen. (Fig. 4, 5)

OCT showed bilateral bulky RNFL which was irregular in uniformity. (Fig. 2, 3)

On angiography, no leakage was seen at the disc margins with foveal auto florescence of the optic disc in both eyes. (Fig. 1) 


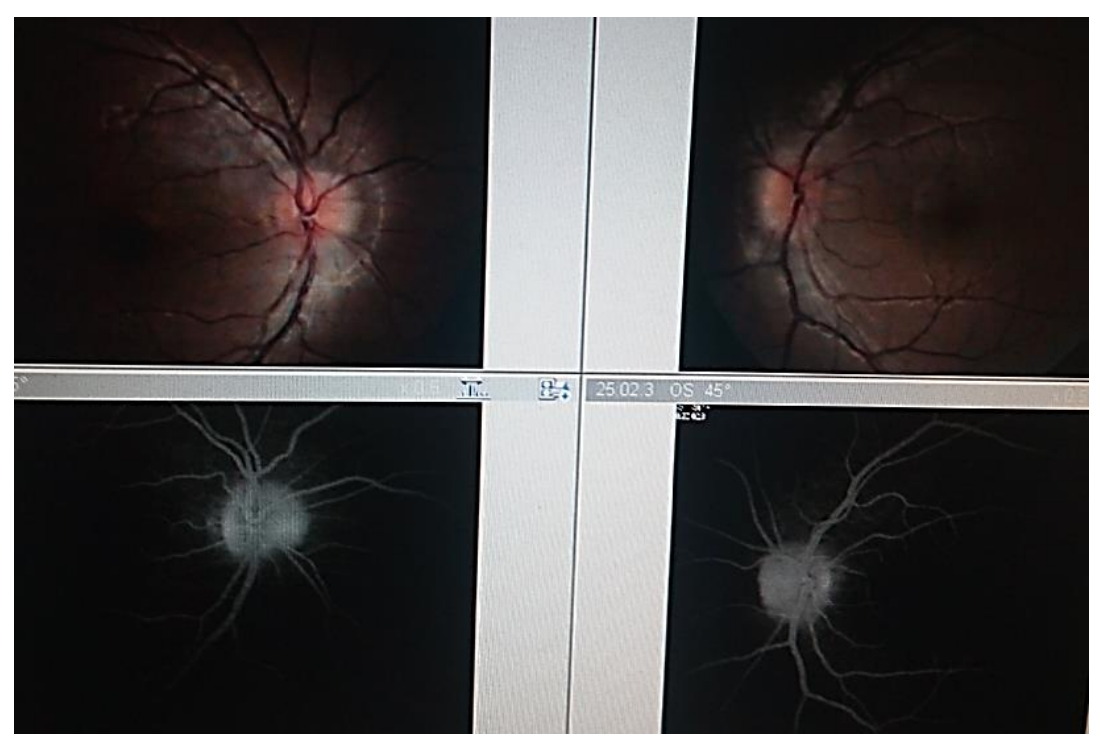

Fig. 1: On FFA, no leakage at the disc margins with auto florescence of the optic disc was seen

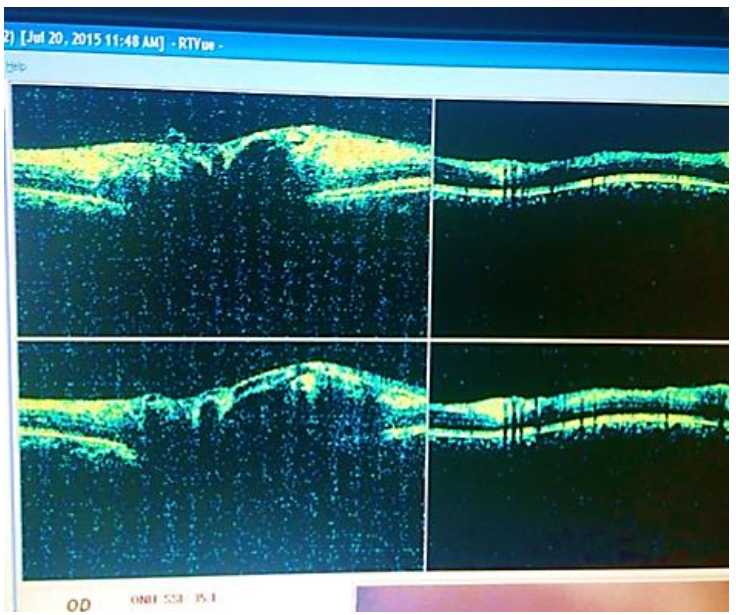

Fig. 2: OCT of right eye showing bulky RNFL which was irregular in uniformity

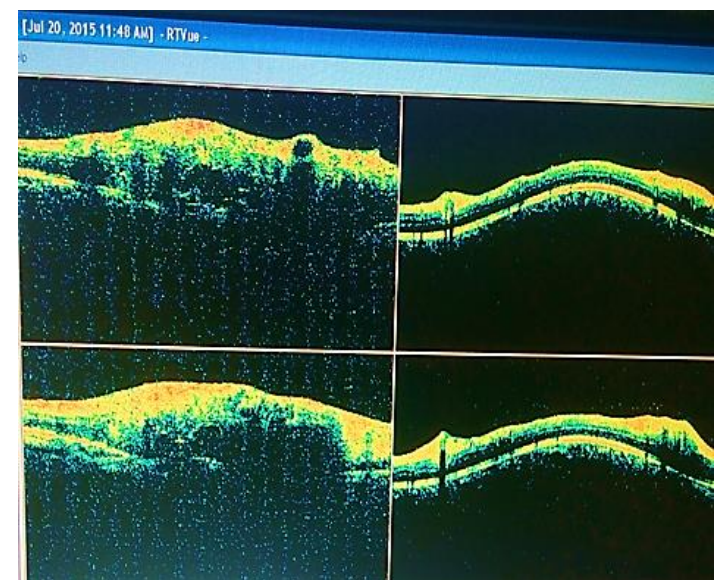

Fig. 3: OCT of left eye showing bulky RNFL which was irregular in uniformity

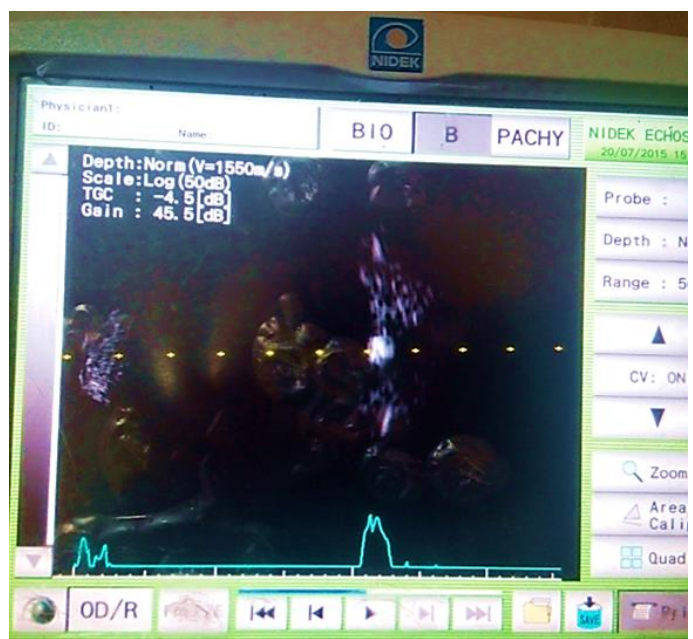

Fig. 4: B SCAN of right eye showing hyper-echoic mass at the optic nerve head

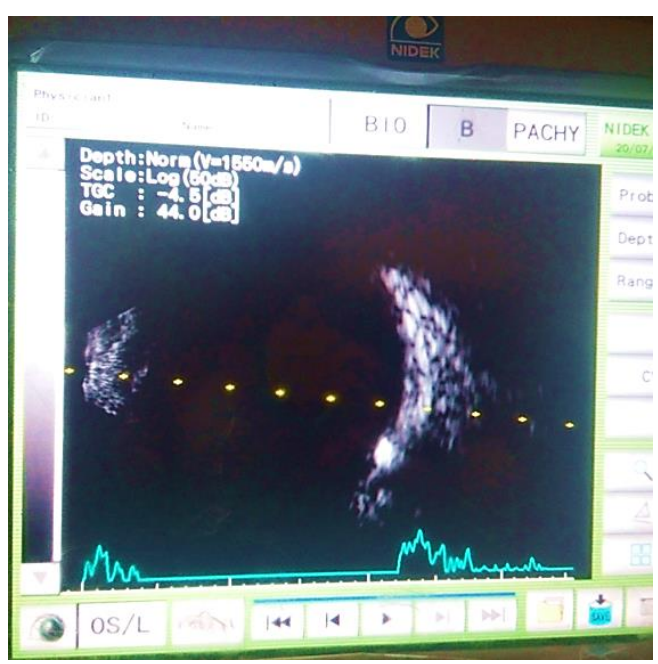

Fig. 5: B SCAN of left eye showing hyper-echoic mass at the optic nerve head 


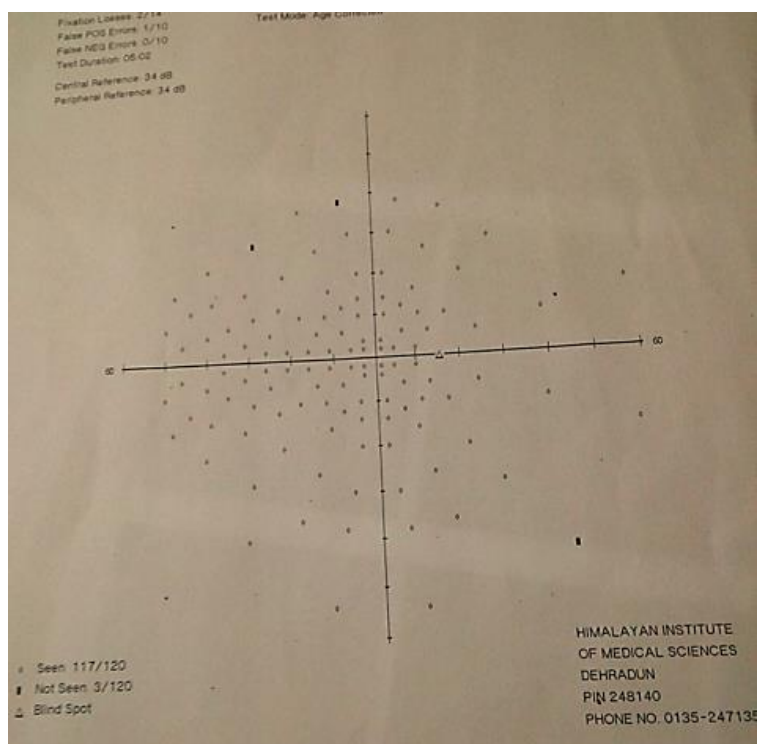

Fig. 6: Normal 120-2 visual fields of right eye

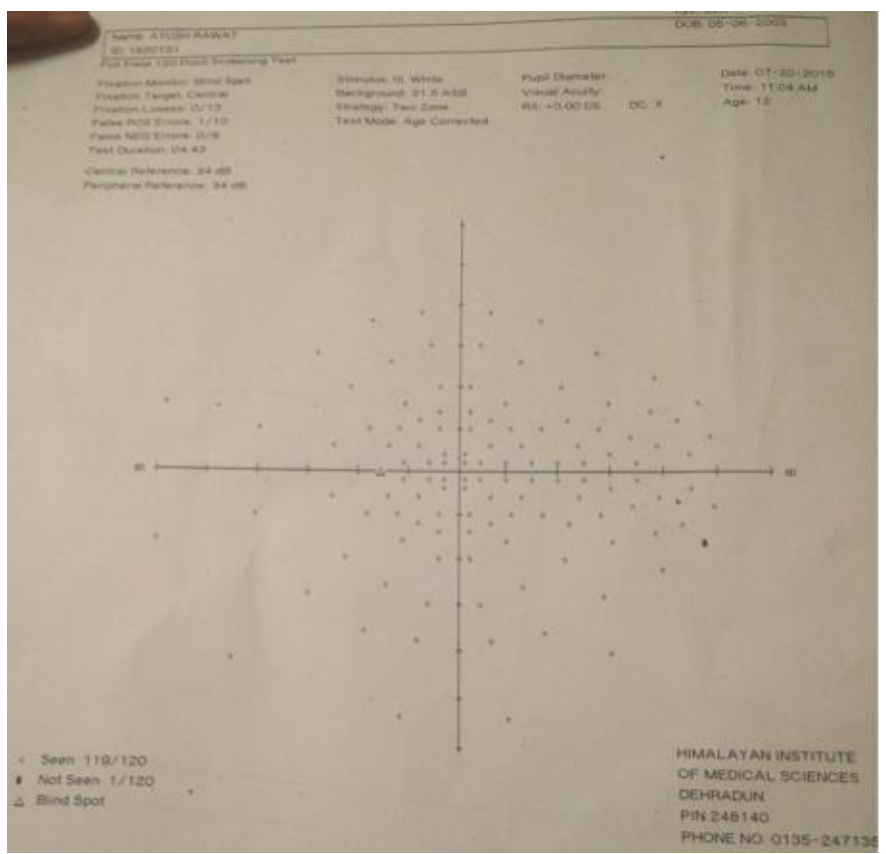

Fig. 7: Normal 120-2 visual fields of left eye

\section{Discussion}

Papilledema because of raised intracranial pressure is associated with swollen peripapillary retina and optic disc hyperemia, which leads to obscuration of retinal vessels at the disc margin. Soft exudates and peripapillary hemorrhages are also likely to occur with papilledema. A spontaneous venous pulsation is absent in papilledema. A severe frontal headache which is occasionally associated with nausea and vomiting is often present. Apart from the difficulties associated with examining young children, accurate imaging and lumbar puncture assessment may require separate anesthetics.
Optic disc drusen are defined as yellow, opalescent, hyaline excrescences derived from calcified axonal debris which are either present on the surface of the optic disc or buried within it. They are bilateral in $70 \%$ of cases with no sex predilection. Disc drusens are inherited in irregular dominant fashion. Its incidence is $0.3 \%$ in general population but increases to $3.4 \%$ in individuals with affected family members. The ophthalmoscopic appearance of optic disc drusen is decided by their location within the optic nerve head. The diagnosis of optic disc drusen is easy in cases with superficial optic disc drusen, but children and young adults are particularly vulnerable to misdiagnosis, such as papilledema because of elevated optic discs due to buried drusen. ${ }^{2}$ 
Drusens are generally considered benign. However, there are some associated complications, the most common being peripheral visual field defects occurring in approximately $75 \%$ of patients. ${ }^{3}$ Central retinal artery occlusion, central vision loss, and anterior ischemic optic neuropathy, juxtapapillary neovascularisation, disc neovascularization are rare potential complications that might occur. ${ }^{4}$ They are often associated with retinitis pigmentosa, angiod streaks and Usher syndrome. Association with pseudoxanthoma elasticum has been reported to be around 1.4 to $3.6 \%$. The incidence of retinal haemorrhages in optic disc drusen has been reported to be $10 \%$. In majority of the patients, optic disc drusens are an incidental finding. ${ }^{5}$

There is no definitive treatment for this condition, but follow-up with an ophthalmologist for serial visual field examinations, optic nerve fiber analysis, and repeat intraocular pressure checks is required. If visual field loss occurs with drusen, medication to decrease the intraocular pressure is started. Laser photocoagulaton should be done in patients threatened central visual acuity. ${ }^{6}$

\section{Conclusion}

Swelling of the optic disc does not always mean raised intracranial pressure, hence other causes must be considered before concluding based on all relevant clinical findings. Importantly, the early diagnosis of ODD spares the patient unnecessary anxiety about intracranial disease and prevents unnecessary interventions. Misdiagnosis may increase the incidence of somatization of these patients, particularly in the young. The ophthalmologist, neurologist, and the reporting radiologist must therefore consider optic disc drusen when dealing with atypical cases of disc swelling.
The main purpose of reporting this case was that a buried optic disc drusen mimics papilloedema and most of the times patients are evaluated further to rule out raised intracranial pressure or tumours. CT scan and MRI of brain is advised and patients unnecessarily have to undergo these procedures. Instead of fancy tests, a basic initial B-scan ultrasonography is helpful in the diagnosis of optic disc drusen.

\section{References}

1. Chiang J, Wong E, Whatham A, et al. The usefulness of multimodal imaging for differentiating pseudopapilloedema and true swelling of the optic nerve head: a review and case series. Clinical and Experimental Optometry. 2015;98(1):12-24.

2. Lorentzen SE (1966) Drusen of the optic disc. Acta Ophthalmol 90:1-180.

3. Beck RW, Corbett JJ, Thompson HS, Sergott RC (1985) Decreased visual acuity from optic disc drusen. Arch Ophthalmol 103:1155.

4. Optic Nerve Head Drusen (2001) Handbook of ocular disease management. Jobson Publishing L.L.C.

5. Golnik K (2006) Congenital anomalies and acquired abnormalities of the optic nerve (version 14.3). Upto Date (On-Line Serial).

6. Davis PL, Jay WM et al (2003) Optic nerve head drusen. Semin Ophthalmol 18(4):222-242. 